Roger Williams University

DOCS@RWU

\title{
The Role of Resilience and Purpose in Life in Habituation to Heat and Cold Pain
}

Bruce W. Smith

University of New Mexico

Erin M. Tooley

Roger Williams University, etooley@rwu.edu

Follow this and additional works at: https://docs.rwu.edu/fcas_fp

Part of the Psychology Commons, and the Public Health Commons

\section{Recommended Citation}

Smith, B.W., Erin M.Tooley, Erica Q. Montague, Amanda E. Robinson, Cynthia J. Cosper and Paul G. Mullins. 2009. "The role of resilience and purpose in life in habituation to heat and cold pain." The Journal of Pain 10 (5): 493-500.

This Article is brought to you for free and open access by the Arts and Sciences at DOCS@RWU. It has been accepted for inclusion in Arts \& Sciences Faculty Publications by an authorized administrator of DOCS@RWU. For more information, please contact mwu@rwu.edu. 


\title{
The Role of Resilience and Purpose in Life in Habituation to Heat and Cold Pain
}

\author{
Bruce W. Smith, ${ }^{\star}$ Erin M. Tooley, ${ }^{\star}$ Erica Q. Montague, ${ }^{\star}$ Amanda E. Robinson, ${ }^{\star}$ \\ Cynthia J. Cosper, ${ }^{*}$ and Paul G. Mullins ${ }^{\dagger}$ \\ *Department of Psychology, University of New Mexico, Albuquerque, New Mexico. \\ ${ }^{\dagger}$ School of Psychology, Bangor University, Bangor, United Kingdom.
}

\begin{abstract}
This study examined the role of resilience in habituation to heat and cold pain in healthy women $(n=47)$. Heat and cold pain thresholds were each assessed across 5 equally spaced trials. Resilience, purpose in life, optimism, social support, and neuroticism were assessed using self-report measures. The hypothesis was that the resilience and the associated resilience factors would be positively related to habituation to heat and cold pain while controlling for neuroticism. Multilevel modeling was used to test the hypothesis. When considering each characteristic separately, resilience and purpose in life predicted greater habituation to heat pain while resilience, purpose in life, optimism, and social support predicted greater habituation to cold pain. When controlling for the other characteristics, both resilience and purpose in life predicted greater habituation to heat and cold pain. Resilience and associated characteristics such as a sense of purpose in life may be related to enhanced habituation to painful stimuli. Future research should further examine the relationship between resilience, purpose in life, and habituation to pain and determine whether psychosocial interventions that target resilience and purpose in life improve habituation and reduce vulnerability to chronic pain.

Perspective: This article showed that resilience and a sense of purpose in life were both related to the ability to habituate to heat and cold pain in healthy women. These personal characteristics may enhance habituation to pain by providing the confidence and motivation to persist in the face of painful stimuli.
\end{abstract}

(C) 2009 by the American Pain Society

Key words: Habituation, resilience, purpose in life, heat pain, cold pain.

T he ability to adapt to pain may play an important role in maintaining quality of life as people age and experience the injuries and illnesses that can cause pain. Although the initial perception of pain serves the critical function of identifying a noxious stimulus, the inability to adapt to repeated nociceptive input may serve no constructive purpose. ${ }^{50}$ The process of adapting to a repetitive stimulus has been referred to as habituation. Whereas sensitization reflects an increase, habitua-

Received August 5, 2008; Revised September 29, 2008; Accepted November 10, 2008.

Supported by a Cross Campus Collaboration grant (\#765110, Bruce W. Smith, Principal Investigator, Paul G.M. Mullins, Co-Principal Investigator) and a Research Allocation Committee grant (\#06-17, Bruce W. Smith, Principal Investigator) from the University of New Mexico, Albuquerque, New Mexico.

Address reprint requests to Bruce W. Smith, PhD, Department of Psychology, University of New Mexico, MSC03 2220, Albuquerque, NM 87131. E-mail: bwsmith@unm.edu

$1526-5900 / \$ 36.00$

(C) 2009 by the American Pain Society

doi:10.1016/j.jpain.2008.11.007 tion has generally been defined as a decreased perception in response to the same stimulus over time. ${ }^{30}$

The ability to habituate to painful stimuli may be important for 2 reasons. First, it may be a protective factor against the development of chronic pain disorders. ${ }^{3} \mathrm{Mi}$ graine headaches and chronic low back pain have been related to the inability to habituate to painful stimuli. ${ }^{13,46}$ Second, the ability to habituate to painful stimuli may be associated with reduced distress and improved functioning in those with chronic pain disorders. The variation in mental health and functioning in pain-related disorders may be partially due to the inability to adapt to repetitive painful stimuli.

Although research has begun to characterize sensitization, especially with regard to temporal summation, ${ }^{10,20,44}$ much less work has focused on habituation. Habituation may involve a different time course than sensitization as well as different mechanisms. ${ }^{21,26}$ There is evidence that habituation may be supported by higher brain regions whose function may involve a variety of 
psychological processes. For example, Bingel, Schoell, Herken, Buchel, and May found neural activation associated with habituation in regions of the brain that have been associated with placebo analgesia and the attentional modulation of pain. ${ }^{3}$ Others have found that habituation may involve the endogenous opioid system, which may be modulated by a variety of psychological processes. $^{24}$

Although it is important to continue to examine the physiology of habituation, it may be vital to also understand habituation from a psychological perspective. Habituation is a general process that may be related to the factors that facilitate psychosocial adaptation. ${ }^{3}$ The decreased perception of pain that characterizes habituation may be related to a more general ability to adapt to stress. If the same psychosocial factors are related to both adaptation to stress and habituation to pain, then interventions that address these factors could be tested to determine whether they also enhance habituation to pain and thereby adaptation to chronic pain.

The process of habituation may be particularly related to the personal characteristic of resilience. Resilience has been defined as the ability to adapt to stressful circumstances and has been strongly related to decreased perceptions of stress. ${ }^{39}$ Resilience also may be important for preventing and living with chronic pain. ${ }^{25}$ It has been related to diminished pain during an ischemic pain induction. ${ }^{16}$ In addition, resilience has been strongly associated with optimism, social support, and a sense of meaning and purpose in life which may also be related to habituation and be possible intervention targets. ${ }^{4,11,45}$ These characteristics have also been related to reduced experimental pain and improved adaptation to chronic pain. $29,40,42$

The purpose of this study was to determine whether resilience and associated factors supporting adaptation to stress would be related to the ability to habituate to painful stimuli. We examined the response to experimental heat and cold pain in healthy women. We assessed resilience and related characteristics including optimism, social support, and a sense of purpose in life. We also assessed neuroticism to control for it as a potentially important vulnerability factor for chronic pain. 5,19 Our hypothesis was that resilience and the associated resilience factors would be related to increased habituation to heat and cold pain stimuli.

\section{Methods}

\section{Participants}

The sample consisted of 47 healthy women. The study was conducted in compliance with the Institutional Review Board of the University of New Mexico and informed consent was obtained by trained research assistants. The participants were recruited through newspaper ads and fliers distributed throughout the community. Only women between the ages of 30 and 60 were recruited for the study. All participants were paid $\$ 20$ for completing the study. All women had average pain ratings of less than 20 on a 0 to 100 scale, had no pain-related diagnoses (eg, fibromyalgia, osteoarthritis, rheumatoid arthritis), and reported that they were not currently depressed or taking antidepressant medications.

The participants were between the ages of 30 and 59 $(\mathrm{M}=48.86, \mathrm{SD}=8.00)$. Fifty-two percent were married, and fewer were never married $(28 \%)$, divorced $(18 \%)$, or widowed (2\%). The majority were Caucasian $(61 \%)$; the rest were Hispanic (32\%) or were of mixed or other ethnicity $(7 \%)$. Most participants had 4 years of college $(65 \%)$ and nearly all had graduated from high school $(93 \%)$. The majority were employed $(74 \%)$ and the mean yearly family income range was $\$ 40,000$ to $\$ 49,000$.

\section{Procedure}

Participants filled out questionnaires that included the measures listed below and were tested for heat and cold pain thresholds using the Contact Heat-Evoked Potential Stimulator (CHEPS; Medoc, Ramat-Yishai, Israel). The CHEPS generates heat or cold sensations using a $3 \times 3-\mathrm{cm}$ contact thermode. This thermode comprises 2 layers: (1) an external layer consisting of a heating foil that has 2 thermocouples (electronic thermal sensors) and (2) a lower layer that is a Peltier element with 1 thermistor (electronic thermal sensor) and 1 water thermistor. The thermode was placed on the thenar (fleshy area of the palm at the base of the thumb) of the right hand.

For the heat pain task, the temperature of the thermode began at $32^{\circ} \mathrm{C}$ and increased $0.5^{\circ} \mathrm{C} / \mathrm{s}$ until the participant indicated that the heat sensation became painful. For the cold pain task, the temperature of the thermode began at $32^{\circ} \mathrm{C}$ and decreased $0.5^{\circ} \mathrm{C} / \mathrm{s}$ until the participant said that the cold sensation became painful. When the participant indicated that the temperature became painful, the researcher pressed a button returning the temperature to $32^{\circ} \mathrm{C}$ in less than 1 second $\left(40^{\circ} \mathrm{C} / \mathrm{s}\right)$. There were 5 consecutive trials for each task and there was a 30-s interval between each trial. The order of presentation of the tasks (eg, heat first, cold second or cold first, heat second) was counterbalanced across participants and there were no order effects.

\section{Measures}

\section{Rationale for the Selection of Measures}

In addition to assessing resilience, we wanted to include psychosocial resources that have been closely identified with resilience. Our criteria for selecting these resources were that they (1) were frequently associated with resilience, (2) represent both personal and social factors, (3) could be targeted in psychological interventions, and (4) have plausible explanations for how they are related to resilience.

We selected optimism and social support because they are often associated with resilience. ${ }^{7,15,23,31}$ In addition, they may be the most frequently studied personal and social resources in models of stress and coping, respectively. ${ }^{1,6}$ Optimism may enhance resilience by making it more likely that people will renew their efforts to attain their goals. ${ }^{36}$ Social support may promote resilience by 
buffering stress and providing emotional and instrumental help in recovering from stressful events. ${ }^{6}$

We also included a sense of meaning and purpose in life because it is almost always included in lists and measures of resilience resources. ${ }^{7,15,43,48}$ A sense of meaning and purpose in life may be critical for surviving and recovering from stressful events. ${ }^{14}$ It may enhance resilience by providing the motivation for recovery following stressful events and has been associated with recovery from knee replacement surgery. ${ }^{40}$

Finally, we assessed neuroticism to control for it as a potentially important vulnerability factor. Neuroticism has been related to pain sensitivity, pain catastrophizing, and the development of chronic pain. ${ }^{5,19}$ In addition, neuroticism has been negatively related to the resilience factors such as optimism and social support and may be important to control for in determining their unique influence on pain. ${ }^{35,41}$

\section{Description of the Measures}

Neuroticism. The tendency to experience negative affects such as anxiety and depression was assessed using the Big Five Inventory. ${ }^{2}$ There were 8 statements (eg, "worries a lot") that were scored on a 5-point scale from 1 = strongly disagree to $5=$ strongly agree. Cronbach's alpha was .768.

Optimism. The tendency to expect positive outcomes was assessed using the Life Orientation Test. ${ }^{35}$ There were 6 items (eg, "I'm always optimistic about my future") that were scored on a 5-point scale from $1=$ strongly disagree to $5=$ strongly agree. Cronbach's alpha was .879.

Purpose in Life. A sense of meaning and purpose in life was assessed using the purpose in life scale from the Scales of Psychological Well-Being. ${ }^{33}$ There were 9 items (eg, "I have a sense of direction and purpose in life") that were scored on a 6-point scale from $1=$ strongly disagree to 6 = strongly agree. Cronbach's alpha was .789 .

Resilience. The ability to adapt to or bounce back from stress was assessed using the Brief Resilience Scale. ${ }^{39}$ There were 6 items (eg, "I tend to bounce back quickly after hard times") that were scored on a 5-point scale from 1 = strongly disagree to $5=$ strongly agree. Cronbach's alpha was .910.

Social Support. The perception that social support is available was assessed using the MOS Social Support Survey. ${ }^{37}$ There were 19 items (eg, "someone who understands your problems") that were scored on a 5-point scale from $1=$ none of the time to $5=$ all of the time. Cronbach's alpha was .982.

\section{Data Analysis Methods}

Multilevel modeling was used to analyze the repeated measures data and test our predictions. This method is useful for the analysis of data that have a nested hierarchical structure. The heat and cold pain threshold data took a hierarchical form, with 5 repeated observations nested within each of the 47 participants. The SPSS 16.0 Mixed program was used for the multilevel analyses and the specifications of the models were based on the guidelines provided by Singer. ${ }^{38}$

The heat and cold pain threshold measures were the criteria variables to be predicted in the analyses. There were 2 basic types of prediction equations in the multilevel analyses: a Level 1 equation, which examined the influence of within-person variations in pain thresholds, and a Level 2 equation, which tested the effects of the resilience factors (eg, resilience, optimism, purpose in life, social support) and neuroticism. In essence, the Level 2 equations address questions regarding between-person differences and take the following form: "Do people who have different scores on a between-persons predictor (eg, resilience) have different levels on the criterion (eg, heat and cold pain thresholds)?"

Level 1 questions address the issue of "when" rather than "who"; for example, "When a person has a highest threshold, is it on an earlier or later trial?" The Level 1 question examined within-person variation with regard to which trial the pain threshold was assessed. To prepare for these analyses, the trial number was centered on each participant by subtracting the average trial number for each participant's from each trial number. The Level 1 equation was specified as follows:

\section{Level 1 : pain threshold ${ }_{i j}=\beta_{0 j}+\beta_{1 j}$ trial number $_{i j}+r_{i j}$}

where pain threshold ${ }_{i j}$ is the pain threshold for participant $\mathrm{i}$ at trial $\mathrm{j}, \beta_{0 \mathrm{j}}$ is the mean pain threshold for participant $\mathrm{j}, \beta_{1 \mathrm{j}}$ is the slope of the trial number predicting the pain threshold for participant $j$, trial number $r_{i j}$ is the trial number of participant $j$ at trial $i$, and $r_{i j}$ is the random component of the pain threshold for participant $j$ at time i.

The main effects of resilience and the resilience factors predicting the mean levels of pain thresholds were modeled at Level 2 so that they could tested and controlled for. Level 2 equations address the question "who" rather than "when." The first Level 2 question addresses "Who has the greatest mean heat (or cold) pain thresholds?" The individual difference variables were used as predictors of variation in the Level 1 pain thresholds. An example of the initial Level 2 equation for this model is a follows:

$$
\text { Level } 2: \beta_{0 j}=\gamma_{00}+\gamma_{01} \text { resilience }+u_{0 j}
$$

where $\beta_{0 \mathrm{j}}$ is the mean pain threshold for participant $\mathrm{j}$, $\gamma_{00}$ is the grand mean pain threshold across all participants, $\gamma_{01}$ is the slope predicting the mean pain threshold for participant $j$ from the resilience score of participant $j$, and $\mathrm{u}_{0 \mathrm{j}}$ is the random component of the mean pain threshold for participant $\mathrm{j}$.

The second Level 2 question addresses "Who has the greatest increase in heat (or decrease in cold) pain thresholds?" Habituation was operationalized as an increase in the heat pain threshold across the 5 heat trials and a decrease in cold pain threshold across the 5 cold trials. Thus, the relationship between the resilience factors and habituation was probed by examining the interaction between these factors and the trial number in 
predicting the pain thresholds. This interaction was specified and modeled as a Level 2 equation as follows:

$$
\text { Level 2: } \beta_{1 j}=\gamma_{10}+\gamma_{11} \text { resilience }+\mu_{1}
$$

where $\beta_{1 \mathrm{j}}$ is the slope of the trial number predicting the pain threshold for participant $j, \gamma_{10}$ is the grand mean for the slope of trial number predicting pain threshold across all participants, $\gamma_{11}$ indicates how the relationship between pain threshold and trial number for participant $\mathrm{j}$ changes as a function of that participant's resilience score, and $\mathrm{u}_{1 \mathrm{j}}$ is the random component of the slope of the trial number predicting the pain threshold for participant $\mathrm{j}$.

The resilience factors (eg, optimism, purpose in life, social support) and neuroticism are not shown but were also modeled as Level 2 predictors (eg, $\gamma_{02}=$ optimism, $\gamma_{03}=$ purpose in life, $\gamma_{04}=$ social support, $\gamma_{05}=$ neuroticism). We also tested the effects of age, income, education, and ethnicity (white vs other) as Level 2 predictors but did not include them in the models because there were no significant main effects and no significant interactions with trial number in predicting either the heat or cold pain thresholds.

Finally, effect sizes were computed for all of the predictors in our hypotheses. According to guidelines outlined in Singer, ${ }^{38}$ effect sizes in multilevel modeling were derived with the variance-covariance parameter estimates. By using these estimates for the intercept, we determined the proportion of explainable between-subjects variance accounted for when resilience and the resilience factors were added to the model.

\section{Results}

Table 1 displays the descriptive statistics and correlations between resilience, resilience factors, and neuroticism. Optimism, purpose in life, and resilience were all positively correlated. Social support was positively correlated with purpose in life and neuroticism was negatively correlated with optimism and resilience.

Fig 1 displays the mean heat pain thresholds across the 5 trials in ${ }^{\circ} \mathrm{C}$ and their standard errors. There was a gradual increase in heat pain thresholds across the 5 trials ( $M=43.29,44.53,45.17,45.53,45.93$, respectively) with an overall mean increase of $2.64{ }^{\circ} \mathrm{C}$. We specified a multilevel model predicting the change in heat pain threshold from the trial number to determine whether the average trial-to-trial increase was significant. The estimate for the fixed effect of the trial number was $.6216(t=16.358, P=$ 000) indicating a significant increase of $.6216^{\circ} \mathrm{C}$ on average for each trial.

Table 2 displays the results for the univariate and multivariate models of resilience, the resilience factors, and neuroticism predicting changes in heat pain thresholds across trials. The estimates are for the interactions between the variable and the trial number. Positive estimates indicate that there was a greater increase in pain thresholds across trials for those higher on the variable as compared with those lower on the variable. Table 2 also shows what would be the estimated slope for a person 1 standard deviation low and for a person 1 standard deviation high on each variable.

In the univariate analyses, resilience, purpose in life, neuroticism were all separately related to changes in heat pain thresholds accounting for $10.27 \%, 1.95 \%$, and $2.11 \%$ of the variance, respectively. Resilience and purpose in life were associated with greater increases in heat pain thresholds across trials. In contrast, neuroticism was associated with smaller increases in heat pain thresholds across trials. There were no significant main effects for resilience, any of the resilience factors, or neuroticism in predicting heat pain thresholds.

For the multivariate analyses, we entered the significant univariate predictors in the equation together and then removed nonsignificant predictors until we had only significant predictors in the equation. As shown in Table 2, the final model included resilience and purpose in life which were still related to increased heat pain thresholds accounting for $9.85 \%$ and $4.55 \%$ of the variance, respectively. This model suggests that a person 1 standard deviation high in resilience would increase an additional $.3979{ }^{\circ} \mathrm{C}$ per trial as compared with a person 1 standard deviation low in resilience ( 8177 minus .4199 from the table). In addition, a person who was 1 standard high vs a person who was 1 standard deviation low on purpose in life would increase an additional $.1322{ }^{\circ} \mathrm{C}$ per trial.

Fig 2 displays the mean cold pain thresholds across the 5 trials in ${ }^{\circ} \mathrm{C}$ and their standard errors. There was a gradual decrease in cold pain thresholds across the trials ( $\mathrm{M}=8.41,8.01,7.28,7.06,6.73$, respectively) with an overall mean decrease of $1.68^{\circ} \mathrm{C}$. We specified a multilevel model predicting the change in cold pain threshold from the trial number to determine whether the average trial-to-trial decrease was significant. The estimate for

Table 1. Descriptive Statistics and Correlations for Resilience, the Resilience Factors, and Neuroticism

\begin{tabular}{|c|c|c|c|c|c|c|c|c|}
\hline & MEAN & $S D$ & RANGE & 1 & 2 & 3 & 4 & 5 \\
\hline Optimism & 4.13 & 0.61 & $2.75-5.00$ & - & & & & \\
\hline Purpose in life & 5.13 & 0.60 & $3.67-6.00$ & $.495^{*}$ & - & & & \\
\hline Resilience & 3.93 & 0.67 & $2.17-5.00$ & $.339 \dagger$ & $.399 *$ & - & & \\
\hline Social support & 4.25 & 0.96 & $1.39-5.00$ & .225 & $.586^{*}$ & .139 & - & \\
\hline Neuroticism & 2.46 & 0.57 & $1.00-3.88$ & $-.332 \dagger$ & -.166 & $-.619 *$ & -.090 & - \\
\hline
\end{tabular}




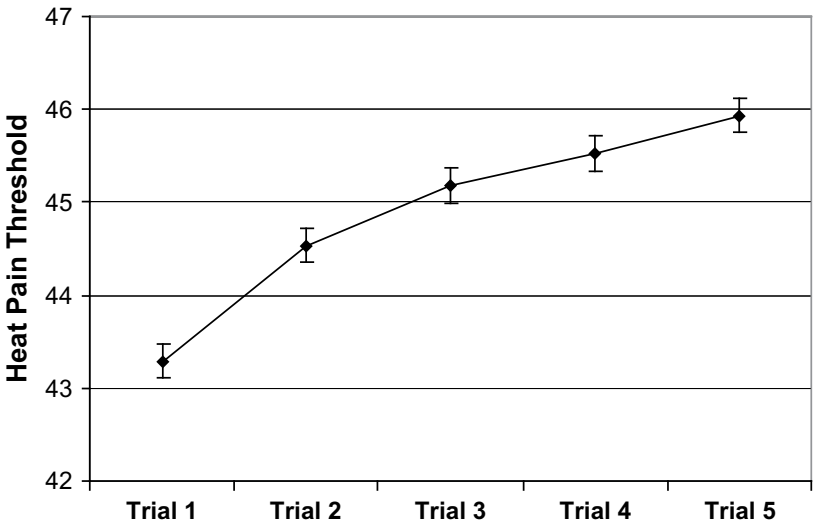

Figure 1. Change in mean heat pain thresholds $\left({ }^{\circ} \mathrm{C}\right)$ across the 5 trials.

the fixed effect of the trial number was $-.4403(t=5.604$, $p=000$ ) indicating a significant decrease of $-.4403^{\circ} \mathrm{C}$ on average for each trial.

Table 3 displays the results for the univariate and multivariate models of resilience, the resilience factors, and neuroticism predicting changes in cold pain thresholds across trials. Again, the estimates are for the interactions between the variable and the trial number. This time, negative estimates indicate that there was a greater decrease in pain thresholds across trials for those higher on the variable as compared with those lower on the variable. Also again, Table 3 shows the estimate slopes for a person 1 standard low and a person 1 standard deviation high on each variable.

In the univariate analyses, purpose in life, social support, optimism, and resilience were all separately related to changes in heat pain thresholds accounting for $11.97 \%, 7.01 \%, 6.46 \%$, and $2.11 \%$ of the variance, respectively. Each of these resilience factors was associated with greater decreases in cold pain thresholds across trials. In contrast, neuroticism was not related to changes in cold pain thresholds. There were no significant main effects for resilience, any of the resilience factors, or neuroticism in predicting cold pain thresholds.

For the multivariate analyses, we entered the significant univariate predictors in the equation together and then removed nonsignificant predictors until there were only significant predictors in the equation. The final model included purpose in life and resilience which were still related to decreases in cold pain thresholds accounting for $12.30 \%$ and $2.03 \%$ of the variance, respectively. This model suggests that a person 1 standard deviation high on purpose in life would decrease an additional $.8156{ }^{\circ} \mathrm{C}$ per trial as compared with a person 1 standard deviation low on purpose in life. In addition, a person 1 standard deviation high vs 1 standard deviation low in resilience would increase an additional $.2219^{\circ} \mathrm{C}$ per trial.

\section{Discussion}

The purpose of this study was to determine whether resilience and associated resilience factors were related to the ability to habituate to experimental heat and cold pain. Based on the conceptual similarity between habituation and the construct of resilience as the ability to adapt to stress, we predicted that resilience would be related to increased habituation. Based on the association between resilience and optimism, social support, and purpose in life, and their effects on experimental and chronic pain, we predicted that these resilience factors would be also be related to increased habituation when controlling for neuroticism.

With regard to heat pain, our hypothesis was supported for resilience and purpose in life in both univariate and multivariate models predicting habituation but was not supported for optimism and social support. With regard to cold pain, our hypothesis was confirmed for all 4 characteristics predicting habituation in univariate models but only for resilience and purpose in life in the multivariate model. Thus, the findings suggest that both resilience and a sense of purpose in life may be related to the ability to habituate to painful stimuli.

Why were resilience and purpose in life related to habituation? The link between these personal characteristics and habituation is consistent with the idea that habituation may be mediated by the brain. Bingel et al. ${ }^{3}$ found neural activation associated with habituation in parts of the brain associated with placebo analgesia and the cognitive modulation of pain. Habituation may be supported by higher brain regions whose function may be affected by behavioral or pharmacological

Table 2. Multilevel Model Predictors of Habituation to Heat Pain Thresholds

\begin{tabular}{|c|c|c|c|c|c|c|c|c|}
\hline & EstimAtE & $S E$ & $d f$ & $t$ & $P$ & $\% V_{A R}$ & $S D$ Low* & $S D H_{I G H}{ }^{*}$ \\
\hline \multicolumn{9}{|c|}{ Univariate predictors } \\
\hline Optimism & .0254 & .0414 & 171.068 & .614 & .540 & - & .6034 & .6342 \\
\hline Purpose in life & .0813 & .0385 & 171.106 & -2.110 & .036 & 1.95 & .5701 & .6675 \\
\hline Resilience & .2558 & .0574 & 172.230 & 4.456 & .000 & 10.27 & .4474 & .7902 \\
\hline Social support & .0506 & .0381 & 171.297 & 1.327 & .186 & - & .5704 & .6672 \\
\hline Neuroticism & -.1573 & .0731 & 171.307 & -2.150 & .033 & 2.11 & .7089 & .5287 \\
\hline \multicolumn{9}{|l|}{ Final model } \\
\hline Purpose in life & .1103 & .0366 & 168.864 & -3.014 & .003 & 4.55 & .5527 & .6849 \\
\hline Resilience & .2969 & .0684 & 169.941 & 4.341 & .000 & 9.85 & .4199 & .8177 \\
\hline
\end{tabular}

*Estimated slopes for the change in heat pain threshold across the trials for a person 1 standard deviation low and a person 1 standard deviation high on each variable, respectively. 


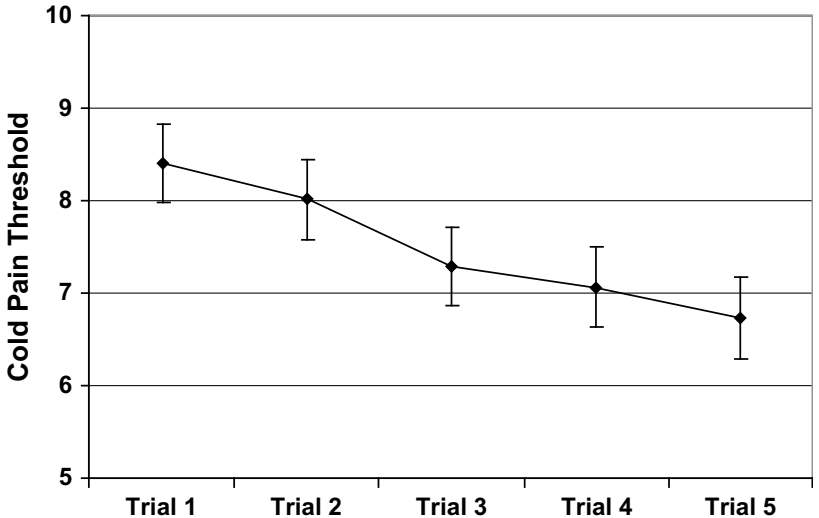

Figure 2. Change in mean cold pain thresholds $\left({ }^{\circ} \mathrm{C}\right)$ across the 5 trials.

interventions that enhance resilience or a sense of meaning and purpose in life. The measure of resilience that we used can be thought of as a measure of self-efficacy with regard to the ability to adapt to stress. The association we found between this self-report measure and habituation suggests that a conscious awareness of this general ability may be linked to a more specific ability to adapt to or habituate to pain.

Surprisingly, our results suggest that a sense of purpose in life may be as strongly related to habituation as resilience itself. This is consistent with the strong role that a sense of purpose in life played in recovery from knee replacement surgery even when optimism was controlled. ${ }^{40}$ Frankl has written extensively on the role that a sense of meaning and purpose may play in the ability to cope with stress. ${ }^{14}$ The motivation that a sense of purpose provides may keep 1 engaged in painful tasks long enough for habituation to take place or even contribute to the development of an enhanced ability to habituate to pain.

What does it mean that neuroticism was not as important as resilience factors in habituation? This is an important question because neuroticism has been related to pain catastrophizing and pain sensitivity. ${ }^{5,19}$ We suspect that the effects of neuroticism relative to the resilience factors may be reduced when there is repeated exposure to painful stimuli. As is key with behavioral approaches to anxiety disorders for which neuroticism is a strong vulnerability factor, repeated exposure may provide evidence that counters the tendency to exaggerate (eg, cata- strophize) about the subsequent painful exposure. ${ }^{8}$ In contrast, resilience and purpose in life may have a steady influence in increasing the self-efficacy and motivation in continuing to expose oneself to painful stimuli.

Why were the responses to heat and cold pain somewhat different? First, there was a greater change in heat pain thresholds across trials $\left(+2.64^{\circ} \mathrm{C}\right)$ than across cold pain thresholds $\left(-1.68^{\circ} \mathrm{C}\right)$. Most of this difference occurred between the first and the second trial (see Figs 1 and 2). We suspect this may have been because heat pain is more easily associated with a burn injury than cold pain and that this fear was allayed after being more cautious on the first trial. Second, there were also some differences in habituation effects for the predictors (see Tables 3and 4). It is possible that the stronger effects for resilience and neuroticism in the heat pain threshold may also be related to a greater fear of burn injury. For example, resilience involves self-efficacy about recovering from a stressful event (eg, burn injury) whereas optimism and purpose in life may have stronger effects when persistence towards a goal alone is important. ${ }^{36,40}$

The next step for future research and clinical interventions regarding habituation may be to determine whether differences in habituation predict the development of chronic pain and to see how much habituation processes may play a role in the ongoing pain and disability of those who already have chronic pain. At this point, it is not clear whether targeting resilience factors that may enhance habituation to acute pain would benefit people with chronic pain. For example, it is possible that chronic pain would reduce resilience and also the ability to habituation to additional pain. If so, resilience-oriented interventions may be doubly important but may also be more difficult or less effective if resilience is reduced below a critical level. Thus, the relationship between resilience, purpose in life, and habituation needs to be studied in those who have or are most at risk for chronic pain disorders.

An important way to advance both research and treatment might be to conduct psychosocial interventions that target either resilience or a sense of purpose in life and observe their effects on habituation to pain. Although it is important not to overestimate the possibility of increasing resilience or purpose in life, there are interventions that may hold promise for doing so. Stress inoculation training $(\mathrm{SIT})^{28}$ has been empirically supported

Table 3. Multilevel Model Predictors of Habituation to Cold Pain Thresholds

\begin{tabular}{|c|c|c|c|c|c|c|c|c|}
\hline & EstimATE & $S E$ & $d f$ & $t$ & $P$ & $\% \operatorname{Var}$ & $S D$ Low* & $S D H_{I G H^{*}}$ \\
\hline \multicolumn{9}{|c|}{ Univariate predictors } \\
\hline Optimism & -.4412 & .1214 & 176.000 & -3.635 & .000 & 6.46 & -.1601 & -.6945 \\
\hline Purpose in life & -.6576 & .1313 & 176.000 & -5.007 & .000 & 11.97 & -.0330 & -.8216 \\
\hline Resilience & -.1646 & .0751 & 176.000 & -2.192 & .030 & 2.11 & -.3170 & -.5376 \\
\hline Social support & -.2934 & .0774 & 176.000 & -3.788 & .000 & 7.01 & -.1465 & -.7081 \\
\hline Neuroticism & .0066 & .1367 & 176.000 & .048 & .962 & - & -.4311 & -.4235 \\
\hline \multicolumn{9}{|l|}{ Final model } \\
\hline Purpose in life & -.6801 & .1346 & 175.000 & -5.055 & .000 & 12.30 & -.0195 & -.8351 \\
\hline Resilience & -.1656 & .0770 & 175.000 & 2.151 & .033 & 2.03 & -.3164 & -.5383 \\
\hline
\end{tabular}

*Estimated slopes for the change in cold pain threshold across the trials for a person 1 standard deviation low and a person 1 standard deviation high on each variable, respectively. 
for a variety of stressors including severe burn injuries and orthopedic surgery. ${ }^{32,49}$ Ross and Berger found that SIT resulted in faster reductions in pain following knee surgery. $^{32}$ The way that SIT employs the learning and rehearsal of cognitive and behavioral techniques for anticipated stressors may be well-suited for boosting resilience.

Similarly, acceptance and commitment therapy ${ }^{22}$ and contextual cognitive-behavioral therapy ${ }^{27}$ for chronic pain use specific techniques for identifying values and goals that may increase a sense of purpose in life. Vowles and McCracken ${ }^{47}$ used an acceptance and values-based approach in chronic pain patients and found that increases in values-based action were related to decreases in pain intensity and pain-related anxiety. Future research could assess changes in resilience and purpose in life in these interventions. If they are important mediators of these changes, then additional studies could determine whether reducing the number of intervention components to those focusing on resilience or purpose in life could increase their efficiency and cost-effectiveness.

This study has several limitations. The pain stimuli were limited to heat and cold, the thenar of the hand, and 1 time interval. It would be useful to vary the time interval and also examine other types of pain and include other parts of the body. Varying the location of the thermode would have made it easier to separate central and peripheral effects. ${ }^{21}$ In addition, we only examined pain threshold and not the response to higher levels of painful stimulation. It is possible that examining pain over a greater range of stimulation could reveal different patterns with regard to habituation. Finally, this study was confined to educated women between the ages of 30 and 60 . We focused on women because they are at greater risk for chronic pain disorders such as fibromyal-

\section{References}

1. Andersson G: The benefits of optimism: A meta-analysis review of the Life Orientation Test. Pers Individ Dif 21: 719-725, 1996

2. Benet-Martinez V, John OP: Los Cinco Grandes across cultures and ethnic groups: Multitrait multimethod analyses of the Big Five in Spanish and English. J Pers Soc Psychol 75: 729-730, 1998

3. Bingel U, Schoell E, Herken W, Buchel C, May A: Habituation to painful stimulation involves the antinociceptive system. Pain 131:21-30, 2007

4. Chamberlain K, Petrie K, Azariah R: The role of optimism and sense of coherence in predicting recovery following knee surgery. Psychol Health 7:201-310, 1992

5. Charles ST, Gatz M, Kato K, Pedersen NL: Physical health 25 years later: The predictive ability of neuroticism. Health Psychol 27:369-378, 2008

6. Cohen S, Wills TA: Stress, social support, and the buffering hypothesis. Psychol Bull 98:310-357, 1985

7. Connor KM, Davidson JRT: Development of a new resilience scale: The Connor-Davidson Resilience Scale (CDRISC). Depression and Anxiety 18:76-82, 2003 gia and rheumatoid arthritis and may have a reduced ability to habituate to pain as compared with men. ${ }^{17}$

However, this study needs to be extended to men and a broader range of age, education, and ethnic backgrounds. Men have reported higher pain thresholds in laboratory research and may be at reduced risk for many clinical pain disorders. ${ }^{12}$ There is some evidence for an age-related decrease in the perception of pain and pain reports even though chronic pain conditions may increase. ${ }^{18}$ African Americans have reported greater pain sensitivity and may experience more pain associated with chronic medical conditions when compared with Caucasians. ${ }^{9}$ There is evidence that individuals with less education may report more chronic and disabling pain. ${ }^{34}$ Thus, future studies could include men, younger and older adults, African Americans, and less educated individuals to determine whether resilience and purpose in life are related to habituation in them and help explain differences in pain due to age, gender, education, and ethnicity.

In conclusion, this study found that the ability to habituate to heat and cold pain was related to resilience and a sense of purpose in life. Thus, the belief that one is capable of adapting to stress may also involve the ability to habituate to pain. In addition, a sense of meaning and purpose in life may provide the motivation for persisting in the face of pain and allowing habituation to take place or even become stronger over time. Future research should examine the relationship between resilience, purpose in life, and habituation in broader samples, identify the processes and mediators that may explain their relationship, and determine whether psychosocial interventions that address resilience and purpose in life result in improved habituation and reduced vulnerability to chronic pain.

8. Craske MG, Barlow DH: Mastery of Your Anxiety and Panic, (4th ed.) New York. NY, Oxford, 2007

9. Edwards $C L$, Fillingam RB, Keefe F: Race, ethnicity, and pain. Pain 94:133-137, 2001

10. Eide PK: Wind-up and the NMDA receptor complex from a clinical perspective. Eur J Pain 2000(4):5-17, 2000

11. Ferreira VM, Sherman AM: The relationship of optimism, pain and social support to well- being in older adults with osteoarthritis. Aging Ment Health 11:89-98, 2007

12. Fillingam RB: Sex-related influences on pain: A review of mechanisms and clinical implications. Rehabil Psychol 48: 165-174, 2003

13. Flor H, Diers M, Birbaumer N: Peripheral and electrocortical responses to painful and non-painful stimulation in chronic pain patients, tension headache patients and healthy controls. Neurosci Lett 361:147-150, 2004

14. Frankl VE: Man's Search for Meaning. New York, NY, Pocket, 1963

15. Friborg $\mathrm{O}$, Hjemdal $\mathrm{O}$, Rosenvinge $\mathrm{JH}$, Martinussen $\mathrm{M}$ : $\mathrm{A}$ new rating scale for adult resilience: What are the central protective resources behind health adjustment? Int J Methods Psychiatr Res 12:65-76, 2003 
16. Friborg $\mathrm{O}$, Hjemdal $\mathrm{O}$, Rosenvinge $\mathrm{JH}$, Martinussen $\mathrm{M}$, Asklaksen PM, Flaten MA: Resilience as a moderator of pain and stress. J Psychosom Res 61:213-219, 2006

17. Ge H, Pascal M, Arendt-Nielsen L: Gender differences in pain modulation evoked by repeated injections of glutamate into the human trapezius muscle. Pain 113:134-140, 2005

18. Gibson SJ, Helme RD: Age-related differences in pain perception and report. Clin Geriatr Med 17:433-456, 2001

19. Goubert L, Cromberz G, Van Damme S: The role of neuroticism, pain catastrophizing and pain-related fear in vigilance to pain: A structural equations approach. Pain 107: 234-241, 2004

20. Granot M, Granovsky Y, Sprecher E, Nir R, Yarnitsky D: Contact heat-evoked temporal summation: Tonic versus repetitive-phasic stimulation. Pain 122:295-305, 2006

21. Greffrath W, Baumgartner U, Treede R: Peripheral and central components of habituation of heat pain perception and evoked potentials in humans. Pain 132:301-311, 2007

22. Hayes SC, Strosahl K, Wilson KG: Acceptance and commitment therapy: An experiential approach to behavior change. New York, NY, Guilford, 1999

23. Horton TV, Wallander JL: Hope and social support as resilience factors against psychological distress of mothers who care for children with chronic physical conditions. Rehabil Psychol 46:382-399, 2001

24. Janicki P, Libich J, Gumulka W: Lack of habituation of pain evoked potentials after naloxone. Pol J Pharmacol Pharm 31:201-205, 1979

25. Karoly P, Ruehlman LS: Psychological "resilience" and its correlates in chronic pain: Findings from a national community sample. Pain 123:90-97, 2006

26. Kleinbohl D, Trojan J, Konrad C, Holzl R: Sensitization and habituation of $\mathrm{AMH}$ and $\mathrm{C}$-fiber related percepts of repetitive radiant heat stimulation. Clin Neurophysiol 117: 118-130, 2006

27. McCracken LM: Contextual Cognitive-Behavioral Therapy for Chronic Pain. Seattle, WA, IASP Press, 2005

28. Meichenbaum D: Stress Inoculation Training. New York, NY, Pergamon, 1985

29. Montoya P, Larbig W, Braun C, Preissel H, Birbaumer N: Influence of social support and emotional context on pain processing and magnetic brain responses in fibromyalgia. Arthritis Rheum 50:4035-4044, 2004

30. Prescott SA: Interactions between depression and facilitation within neural networks: Updating the dual-process theory of plasticity. Learn Mem 5:446-466, 1998

31. Riolli L, Savick V, Cepani A: Resilience in the face of catastrophe: Optimism, personality and coping in the Kosovo crisis. J Appl Soc Psychol 32:1604-1627, 2002

32. Ross MJ, Berger RS: Effects of stress inoculation training on athletes' postsurgical pain and rehabilitation after orthopedic injury. J Consult Clin Psychol 64:406-410, 1996

33. Ryff $C D$, Keyes CLM: The structure of psychological wellbeing revisited. J Pers Soc Psychol 69:719-727, 1995
34. Saastamoinen $\mathrm{P}$, Leino-Arjas $\mathrm{P}$, Laaksonen $\mathrm{M}$, Lahelma $\mathrm{E}$ : Socio-economic differences in the prevalence of acute, chronic, and disabling chronic pain among ageing employees. Pain 114:364-371, 2005

35. Scheier MF, Carver CS, Bridges MW: Distinguishing optimism from neuroticism (and trait anxiety, self-mastery, and self-esteem): A reevaluation of the Life Orientation Test. J Pers Soc Psychol 67:1063-1078, 1994

36. Scheier MF, Weintraub JK, Carver CS: Coping with stress: Divergent strategies of optimists and pessimists. J Pers Soc Psychol 51:1257-1264, 1986

37. Sherbourne CD, Stewart AL: The MOS social support survey. Soc Sci Med 32:705-714, 1991

38. Singer JD: Using SAS PROC MIXED to fit multilevel models, hierarchical models, and individual growth models. J Educ Beh Stat 24:323-355, 1998

39. Smith BW, Dalen J, Wiggins K, Tooley E, Christopher P, Bernard J: The Brief Resilience Scale: Assessing the ability to bounce back. Int J Behav Med 15:294-200, 2008

40. Smith BW, Zautra AJ: The role of purpose in life in recovery from knee surgery. Int J Behav Med 11:197-202, 2004

41. Smith TW, Pope MK, Rhodewalk F, Poulton JL: Optimism, neuroticism, coping, and symptom reports: An alternative interpretation of the Life Orientation Test. J Pers Soc Psychol 56:640-648, 1989

42. Snyder CR, Berg C, Woodward JT, Gum A, Rand KL, Wrobleski KK, Brown J, Hackman A: Hope against the cold: Individual differences in trait hope and acute pain tolerance on the cold pressor task. J Pers 73:287-312, 2005

43. Southwick SM, Vythilingam M, Charney DS: The psychobiology of depression and resilience to stress: Implications for prevention and treatment. Annu Rev Clin Psychol 1:255-291, 2005

44. Staud R, Craggs JG, Robinson ME, Perlstein WM, Price DD: Brain activity related to temporal summation of C-fiber evoked pain. Pain 129:130-142, 2007

45. Treharne GJ, Kitas GD, Lyons AC: Well-being in rheumatoid arthritis: The effects of disease direction and psychosocial factors. J Health Psychol 10:457-474, 2005

46. Valeriani $M$, de Tommaso $M$, Restuccia $D$, Le Pera $D_{\text {, }}$ Guido M, lannetti GD, Libro G, Truini G, Di Trapani G, Puca F, Tonali R, Cruccu G: Reduced habituation to experimental pain in migraine patients: $\mathrm{A} \mathrm{CO}_{2}$ laser evoked potential study. Pain 105:57-64, 2003

47. Vowles KE, McCracken LM: Acceptance and valuesbased action in chronic pain: A study of treatment effectiveness and process. J Consult Clin Psychol 76:397-407, 2008

48. Wagnild GM, Young HM: Development and psychometric evaluation of the resilience scale. J Nurs Meas 1:165-178, 1993

49. Wernick RL, Jaremko ME, Taylor PW: Pain management in severely burned adults: A test of stress inoculation. J Beh Med 4:103-109, 1981

50. Woolf CJ, Ma Q: Nociceptors-noxious stimulus detectors. Neuron 55:353-364, 1998 